\title{
Reproducibility of native and contrast-enhanced CMR techniques to measure lesion size following acute myocardial infarction
}

\author{
Enver Tahir ${ }^{1 *}$, Martin R Sinn ${ }^{1}$, Maxim Avanesov ${ }^{3}$, Joshua Wien ${ }^{4}$, Dennis Saering ${ }^{3}$, Christian Stehning ${ }^{4}$, \\ Ulf K Radunski ${ }^{2}$, Kai Muellerleile², Gerhard Adam', Gunnar Lund ${ }^{1}$ \\ From 19th Annual SCMR Scientific Sessions \\ Los Angeles, CA, USA. 27-30 January 2016
}

\section{Background}

The purpose of this study was to analyze the reproducibility of native and contrast-enhanced CMR techniques to measure lesion size after acute myocardial infarction (AMI) using native T1/T2 mapping, T2-weighted (T2w) imaging, contrast-enhanced late gadolinium enhancement (LGE), post-contrast T1 mapping and extracellular volume (ECV) quantification.

\section{Methods}

Lesion size was independently quantified by 2 experienced observers in total of 120 consecutive CMRs obtained in 30 patients within the first 6 months after AMI using native and contrast-enhanced sequences. Lesion sizes were quantified using a threshold method (cutoff $>2$ SD of remote normal myocardium) on basal, midventricular and apical short-axis left ventricular slices. Lesion size is given as the mean of both observers. Bland-Altman analysis was performed to determine the agreement between the two observers. Non-parametric Levene's test was used to compare the variances of the relative differences. Statistical analysis was performed using GraphPad Prism 6.

\section{Results}

The relative median difference of the native CMR techniques were $-1.95 \%(-12.7 \%$ and $9.8 \%)$ for $\mathrm{T} 2 \mathrm{w},-5.3 \%$ $(-19.6 \%$ and $14.8 \%)$ for native T1 and $-4.0 \%(-23.9 \%$ and 9.9\%) for native T2 (Fig. 1). Results for contrast-enhanced CMR imaging were: $2.9 \%(-4.5 \%$ and $10.5 \%)$ for LGE, $7.5 \%(-2.4 \%$ and $21.5 \%)$ for post-contrast $\mathrm{T} 1$ and $-2.9 \%$ $(-11.4 \%$ and $9 \%)$ for ECV measurement. Bland Altman analysis revealed a better agreement for all post-contrast sequences indicted by lower limits of agreement compared to native sequences (Figure 1). The increased variability of native imaging was caused by higher interobserver differences in small lesions with sizes between 0-15 \%LV compared to lager lesions $>15 \% \mathrm{LV}$. This bias was not observed for post-contrast imaging.

\section{Conclusions}

In general, there was a good agreement between the two observers to measure lesion size after AMI, but all postcontrast sequences had a better agreement compared to the native sequences. The low agreement of native imaging was mainly caused by higher interobserver differences in small lesions with lesion sizes between 0-15 \%LV compared to lager lesions $>15 \% \mathrm{LV}$.

\section{Authors' details}

'Diagnostic and Interventional Radiology, University Hospital Eppendorf, Hamburg, Germany. ${ }^{2}$ General and Interventional Cardiology, University Medical Center Hamburg-Eppendorf, Hamburg, Germany. ${ }^{3}$ Institute of Applied Sciences, Wedel, Germany. ${ }^{4}$ Phillips Research Laboratory, Hamburg, Germany.

Published: 27 January 2016

doi:10.1186/1532-429X-18-S1-P92

Cite this article as: Tahir et al:: Reproducibility of native and contrastenhanced CMR techniques to measure lesion size following acute myocardial infarction. Journal of Cardiovascular Magnetic Resonance 2016 18(Suppl 1):P92. 


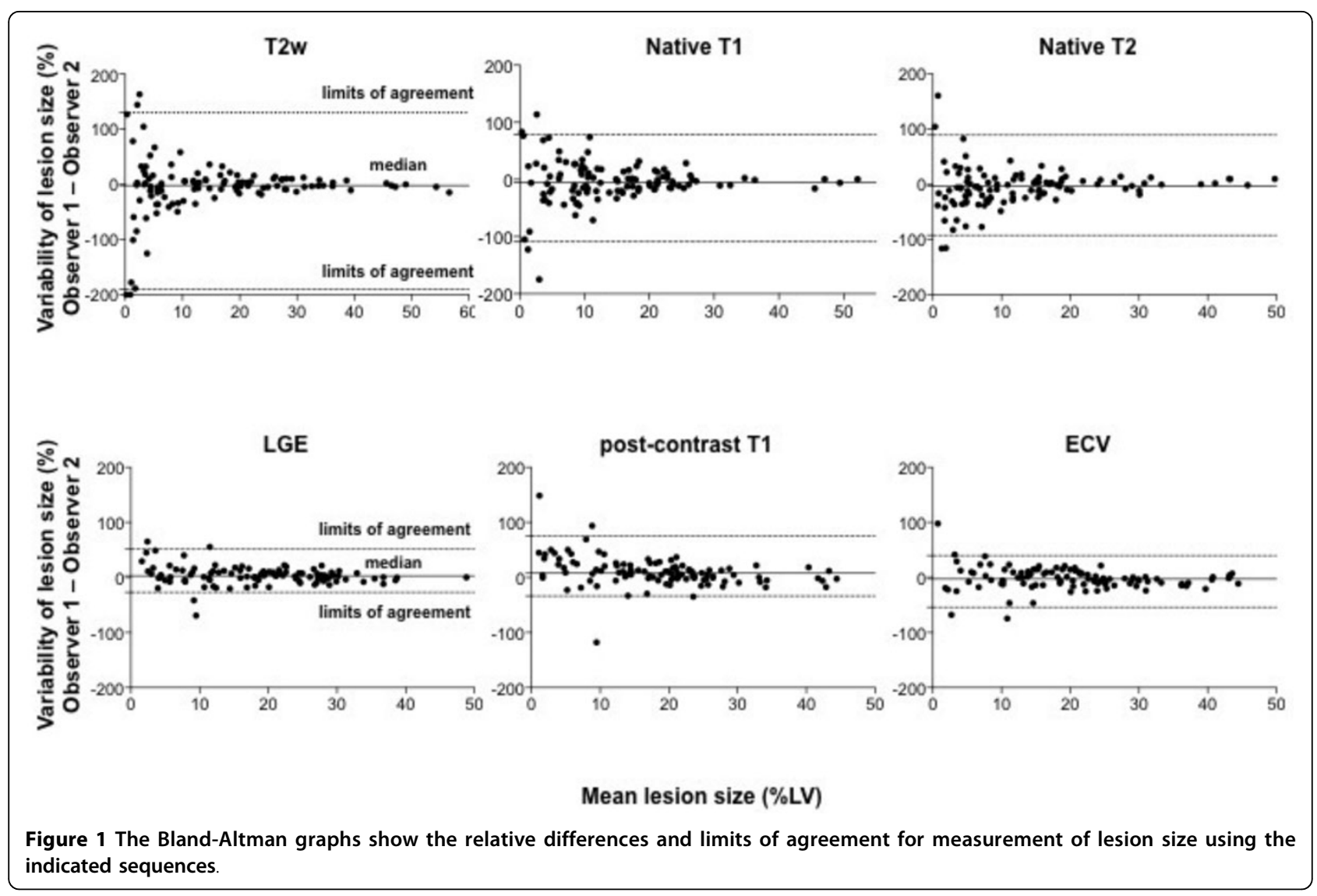

